\title{
Measurement and finite element analysis of the load-dependent pressure redistribution behavior of various types of mattresses
}

\begin{abstract}
Load dependence must be kept in mind when evaluating mattress pressure redistribution, to prevent the development of pressure ulcers at bony prominences. However, there is no standardized method for analyzing load-dependent behavior after mattress pressure redistribution. In this study, a portable palmtop device with a simple load-sensing mechanism was developed for measuring the mean pressure exerted by a protruding shaft surrounded by a disc on low-resilience polyurethane ( $\mathrm{LRPu}$ ) foam, latex foam, coconut fiber and latex (CFL) mattresses, as well as polyurethane $(\mathrm{Pu})$ foam bed and an LRPu foam mattress laid on a bed. Finite element (FE) analysis was used to analyze deformation and contact pressure in detail. The pressure redistribution was greatest for the LRPu foam mattress, and excessive compression was avoided by using an underlay made of stiff Pu foam. FE analysis revealed that the contact pressure increased significantly near the outer circumference of the protruding shaft and the surrounding disc. Significant nonuniformity in pressure was evident, according to the edge and bottom geometry of the device. The measurements and FE analysis revealed load-dependent pressure redistribution behavior, which should allow mattresses to be tailored on an individual basis.
\end{abstract}

Keyword: Finite element method; Mattress; Pressure redistribution; Pressure ulcer; Pressure redistribution measurement device; Support surface 